\title{
Squark/gluino searches in hadronic channels with ATLAS
}

\author{
Antonia STRÜBIG* on behalf of the ATLAS collaboration \\ Nikhef National institute for subatomic physics (NL) \\ E-mail: antonia.struebig@cern.ch
}

This document presents recent results of supersymmetry searches at ATLAS with $3.2 \mathrm{fb}^{-1}$ of proton-proton collisions at the LHC with $\sqrt{s}=13 \mathrm{TeV}$. The presented searches focus on Rparity conserving models with strong production of squarks and gluinos, the dominant production process for early $\sqrt{s}=13 \mathrm{TeV}$ LHC running. Covered final states include jets, missing transverse momentum, b-jets and photons. No significant excess is observed and upper limits on the expected number of BSM events and exclusion limits on the squark and gluino masses for each model are presented.

Fourth Annual Large Hadron Collider Physics

13-18 June 2016

Lund, Sweden

${ }^{*}$ Speaker. 


\section{Introduction}

Supersymmetry (SUSY) is a popular extension of the Standard Model of particle physics (SM). It postulates a superpartner with spin altered by $\frac{1}{2}$ for every existing SM particle. R-parity conserving models require that SUSY particles are produced in pairs and in addition the lightest SUSY particle (LSP) is stable, providing a promising dark matter candidate. In many models the lightest Neutralino $\widetilde{\chi}_{1}^{0}$ is the LSP. In models with a general gauge mediated SUSY breaking mechanism the LSP is the Gravitino $\widetilde{G}$.

For Run II of the LHC the center-of-mass energy was increased from $\sqrt{s}=8 \mathrm{TeV}$ to $13 \mathrm{TeV}$. As shown in figure 1 the strong production cross section is several magnitudes larger than the one for electroweak SUSY production.

The decay of the produced squarks and gluinos leads to a final state with quarks and the LSP resulting in high transverse momentum jets and missing transverse energy, respectively. For simplicity only models with direct decay to the LSP or a maximum of two intermediate steps are studied.

This document presents several ATLAS [1] SUSY searches carried out with $3.2 \mathrm{fb}^{-1}$ of data of proton-proton collisions with $\sqrt{s}=13 \mathrm{TeV}$ collected at the LHC in 2016. The presented analyses all focus on the dominant strong SUSY production leading to high $\mathrm{p}_{\mathrm{T}}$ jets and $\mathrm{E}_{\mathrm{T}}^{\mathrm{miss}}$ in the final state.

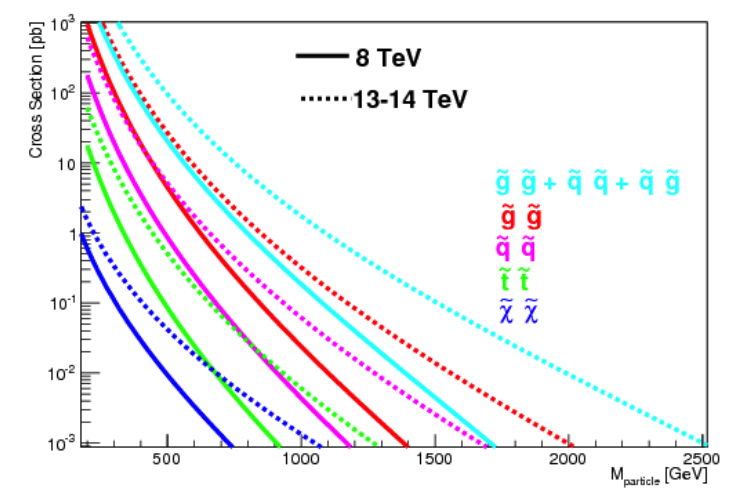

Figure 1: Cross sections for SUSY particle production, the production cross section for squarks and gluinos is shown for $\sqrt{s}=8$ and $13 \mathrm{TeV}$. The electroweak production cross section is shown for $\sqrt{s}=8$ and 14 TeV. [2]

\section{2-6 jets search}

The ATLAS search for squarks and gluinos in final states with jets and missing transverse momentum [3] is motivated by the large number of R-parity conserving SUSY models. This search considers different simplified SUSY models. In the first two models the strongly produced squarks and gluinos directly decay to a quark + LSP and $q \bar{q}$ pair + LSP respectively. The third considered model is a 'one-step' decay model, where the gluino decays via a $q \bar{q}$ pair $+\widetilde{\chi}_{2}^{ \pm}$and further to a $\mathrm{W}$ boson and the LSP producing more jets in the final state.

The search uses seven inclusive signal regions (SR) characterised by the number of jets in the final 
state. The selection criteria for each SR are optimized for the expected signal significance. All signal regions are defined by a veto on any leptons in the final state. The main discriminating variable between signal and background is the effective mass $m_{\text {eff }}$ defined as the scalar sum of the missing transverse momentum and the transverse momenta of the jets, $m_{\mathrm{eff}}:=E_{\mathrm{T}}^{\mathrm{miss}}+\sum_{i} p_{\mathrm{T}}^{\mathrm{jet}_{i}}$. As additional selection the minimal azimuthal separation between $E_{\mathrm{T}}^{\mathrm{miss}}$ and the transverse momentum of the jets, $\Delta \Phi\left(\right.$ jet, $\left.E_{\mathrm{T}}^{\text {miss }}\right)$, is used. Together with a selection applied on the ratio $E_{\mathrm{T}}^{\mathrm{miss}} / \mathrm{m}_{\mathrm{eff}}$ it helps to reduce background from multi-jet processes. In the two-jet SRs $E_{\mathrm{T}}^{\mathrm{miss}} / \mathrm{m}_{\text {eff }}$ is replaced by $E_{\mathrm{T}}^{\text {miss }} / \sqrt{H_{\mathrm{T}}}$ with $H_{\mathrm{T}}$ the scalar sum of the transverse jet momenta.

All SR require $E_{\mathrm{T}}^{\text {miss }}$ larger than $200 \mathrm{GeV}$ and a leading jet $\mathrm{p}_{\mathrm{T}}$ of at least $200 \mathrm{GeV}$. The selection on $m_{\text {eff }}$ ranges from $1200 \mathrm{GeV}$ for the loosest two-jet SR to $2000 \mathrm{GeV}$ in the four-jet region. The different jet-multiplicities target different scenarios. The SRs requiring at least two jets are aimed at squark-pair production with the direct decay of the squark to the LSP and at least one jet, e.g. $\widetilde{q} \rightarrow q \widetilde{\chi}_{1}^{0}$. Direct gluino decay typically leads to two jets, e.g. $\widetilde{g} \rightarrow q \bar{q} \widetilde{\chi}_{1}^{0}$ and therefore gluino-pair production is targeted by the SR requiring at least four jets. The SRs with more than five jets are targeting longer decay chains with more jets in the final state.

For each SR a number of orthogonal control regions (CR) is defined to estimate the background in that SR. The estimation for the different background sources from the CR is then used to normalize the Monte Carlo simulation. The main background sources for this analysis are: Z+jets, W+jets, top quark pairs and single top quarks, dibosons and multi-jet production. The multi-jet background is negligible after the requirements on $\Delta \Phi\left(\mathrm{jet}, E_{\mathrm{T}}^{\mathrm{miss}}\right)$ and $E_{\mathrm{T}}^{\text {miss }} / \mathrm{m}_{\mathrm{eff}}$. For the estimation of the diboson production a MC simulation normalized to the NLO cross-section is used. The other backgrounds are estimated with help of the dedicated CRs for each background and the predictions are cross-checked in validation regions (VR). The selection criteria for the VRs are distinct from the corresponding $\mathrm{CR}$ and at the same time keeping the probability of signal contamination at a minimum.

The results are gained by using a background-only likelihood fit with the observed event yields from the CRs as only constraint for each SR. If no excess is observed a model-independent fit is conducted to set limits on the visible Beyond the Standard Model (BSM) cross section in each SR. This fit additionally contains the observed number of events in the SR as input.

For the model dependent limits on the squark (gluino) and neutralino masses a model-dependent fit is used including the yield in the SR and also the signal contamination in the CR on top of the model-independent fit. The results from the model-dependent fit of each SR are used to set limits on specific SUSY models. For each point in the model's parameter space the SR with the highest expected signal sensitivity is used.

There was no significant excess observed and the results agree well with the SM predictions. Figure 2 shows the limit for the strong production of squarks (gluinos) with direct decay to quarks and the LSP. For a massless lightest neutralino squark and gluino masses below $1.03 \mathrm{TeV}$ and $1.51 \mathrm{TeV}$ are excluded respectively. The limit for a gluino decaying via an intermediate $\tilde{\chi}_{2}^{ \pm}$to the LSP is shown in figure 3 . For this model and a lightest neutralino mass of $\sim 200 \mathrm{GeV}$ gluino masses below $1.5 \mathrm{TeV}$ are excluded at $95 \%$ confidence level. 

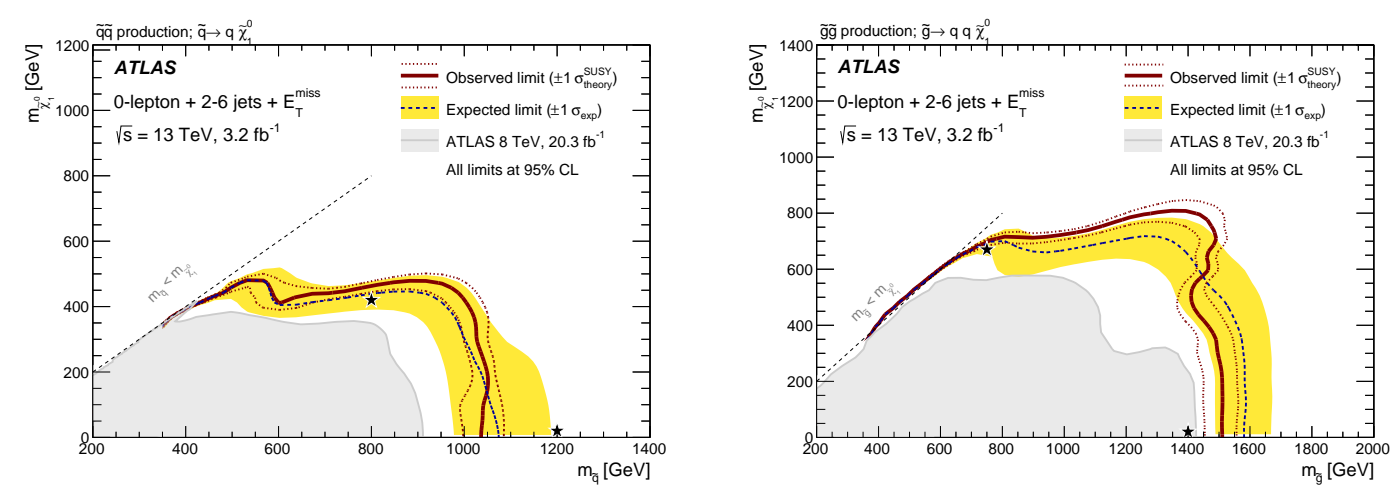

Figure 2: Exclusion limits for direct production of light-flavor squark pairs with decoupled gluinos (left) and gluino pairs with decoupled squarks (right). Gluinos (light-flavor squarks) are required to decay to two quarks (one quark) and a neutralino LSP. Exclusion limits are obtained by using the signal region with the best expected sensitivity at each point. The blue dashed lines show the expected limits at 95\% CL, with the light (yellow) bands indicating the $1 \sigma$ excursions due to experimental and background-only theoretical uncertainties. Observed limits are indicated by medium dark (maroon) curves where the solid contour represents the nominal limit, and the dotted lines are obtained by varying the signal cross-section by the renormalization and factorization scale and PDF uncertainties. [3]

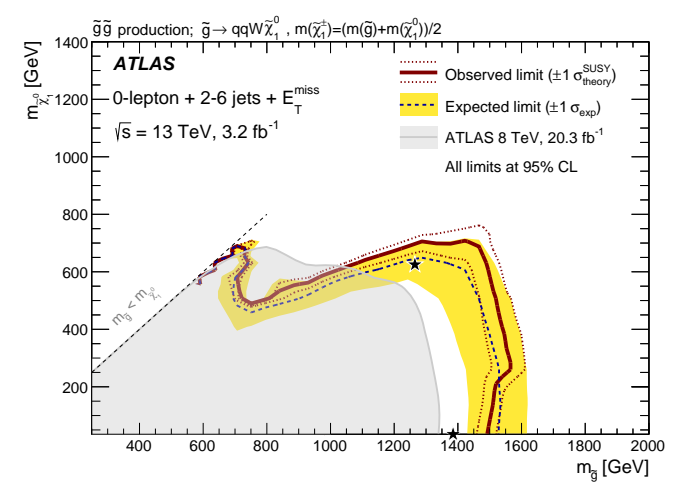

Figure 3: Exclusion limits for pair-produced gluinos each decaying via an intermediate $\widetilde{\chi}_{2}^{ \pm}$to two quarks, a $\mathrm{W}$ boson and a $\widetilde{\chi}_{1}^{0}$ for models with a fixed $\mathrm{m}\left(\widetilde{\chi}_{2}^{ \pm}\right)=\left(\mathrm{m}(\widetilde{g})+\mathrm{m}\left(\widetilde{\chi}_{1}^{0}\right)\right) / 2$ and varying values of $\mathrm{m}(\widetilde{g})$ and $\mathrm{m}\left(\widetilde{\chi}_{1}^{0}\right)$. Exclusion limits are obtained by using the signal region with the best expected sensitivity at each point. The blue dashed lines show the expected limits at 95\% CL, with the light (yellow) bands indicating the $1 \sigma$ excursions due to experimental and background-only theoretical uncertainties. Observed limits are indicated by medium dark (maroon) curves where the solid contour represents the nominal limit, and the dotted lines are obtained by varying the signal cross-section by the renormalization and factorization scale and PDF uncertainties. [3]

\section{Multi-jets search}

The ATLAS search for new phenomena in final states with large jet multiplicities and transverse missing momenta [4] is targeting cascade decays of new particles where the multiple step decays provide the many hadronic jet seeds. There are several SUSY models leading to this scenario. The results of this search are evaluated in two different type of models. One is a simplified model for gluino-pair production with a '2-step' decay of the gluino via $q \bar{q}$ and a $\widetilde{\chi}_{2}^{ \pm}$to a W-boson and a $\widetilde{\chi}_{2}^{0}$ which further decays to a Z-boson and the lightest neutralino. The other model belongs 
to the 19 free parameter space of the phenomenological Minimal Supersymmetric Standard Model (pMSSM). The parameter set is chosen in a way that only the gluinos, the neutralinos and the chargino are kinematically accessible. The gluino decays dominantly to top and bottom quarks when the mass difference between gluino and chargino is sufficiently large. This results in several b-jets and relatively low $E_{\mathrm{T}}^{\text {miss }}$ causing other hadronic searches to be quite insensitive to these type of models.

The search employs 15 inclusive signal regions which all place a veto on events containing leptons. There are two sets, one requiring all signal jets to have a $\mathrm{p}_{\mathrm{T}}$ larger than $50 \mathrm{GeV}$, the other requiring a jet $\mathrm{p}_{\mathrm{T}}$ above $80 \mathrm{GeV}$. The $50 \mathrm{GeV}$ set includes SRs requiring at least eight, nine or ten jets, the $80 \mathrm{GeV}$ set has requirements for at least seven or eight jets. All SRs are binned in the number of b-jets, selecting a minimum of zero, one or at least two b-jets. The main discriminating variable between signal and background is $E_{\mathrm{T}}^{\text {miss }} / \sqrt{H_{\mathrm{T}}}$ with $H_{\mathrm{T}}$ the scalar sum of all transverse jet momenta above $40 \mathrm{GeV}$. All SRs only select events with a $E_{\mathrm{T}}^{\text {miss }} / \sqrt{H_{\mathrm{T}}}>4 \mathrm{GeV}^{1 / 2}$.

The two main background contributions for this analysis are multijet production, both from strong interaction processes and fully hadronically decaying $t \bar{t}$, and leptonic backgrounds, including partially leptonically decaying $t \bar{t}$ and leptonically decaying $\mathrm{W}$ and $\mathrm{Z}$ bosons in association with jets. The estimation of the minor contributions to the leptonic backgrounds are taken directly from MC simulation. The dominant leptonic contributions by $t \bar{t}$ and $\mathrm{W}$ boson production are estimated via a simultaneous fit of the corresponding CRs. The multijet estimation is derived directly from the data. The main discriminating variable $E_{\mathrm{T}}^{\text {miss }} / \sqrt{H_{\mathrm{T}}}$ is invariant under jet multiplicity therefore the estimation can be taken from a CR with lower jet multiplicities than the SR. The $E_{\mathrm{T}}^{\text {miss }} / \sqrt{H_{\mathrm{T}}}$ distribution template is then normalized to data in a second CR with the same jet multiplicity but much lower requirement on $E_{\mathrm{T}}^{\text {miss }} / \sqrt{H_{\mathrm{T}}}$ than in the SR. The templates are produced separately for each $\mathrm{SR}$ and b-jet requirement and cross-checked in VRs with lower jet multiplicities and or $E_{\mathrm{T}}^{\text {miss }} / \sqrt{H_{\mathrm{T}}}$. There is no significant excess above the SM observed. Model-independent limits on the expected number of BSM signal events as well as exclusion limits in the models' phase spaces are produced as described in 2. Figure 4 shows the exclusion limit for the '2-step' and the 'pMSSM' model, in both cases gluino masses below $\sim 1400 \mathrm{TeV}$ can be excluded with $95 \%$ confidence level.

\section{4. b-jets search}

The ATLAS search for pair production of gluinos decaying via stop and sbottom in events with b-jets and large missing transverse momentum [5] is aimed at gluino-mediated production of stop and sbottom squarks. The solution to the hierarchy problem offered by SUSY requires light third generation quarks to prevent unnatural fine-tuning in the Higgs sector. Both should be close to the weak scale, with the gluino mass expected to be around the TeV scale this type of model is well motivated. In the simplified 'Gtt' ('Gbb') model the gluino decays via an off-shell $\widetilde{t}(\widetilde{b})$ to two top (bottom) quarks and the lightest neutralino. Both models have four b-jets in the final state with four additional $\mathrm{W}$ bosons in the case of the Gtt model.

The search employs a total of eight SRs. For the Gbb model there are three SRs all vetoing any signal leptons and requiring at least four jets of which three must be b-tagged. To reduce multijet background only events with a minimum $\Delta \Phi\left(\right.$ jet,$\left.E_{\mathrm{T}}^{\text {miss }}\right)>0.4$ are selected. The requirements on $E_{\mathrm{T}}^{\text {miss }}$, the leading jet $\mathrm{p}_{T}$ and $m_{\text {eff }}^{4 \text { leading jets }}$ are varied, targeting low, moderate and high mass splitting 

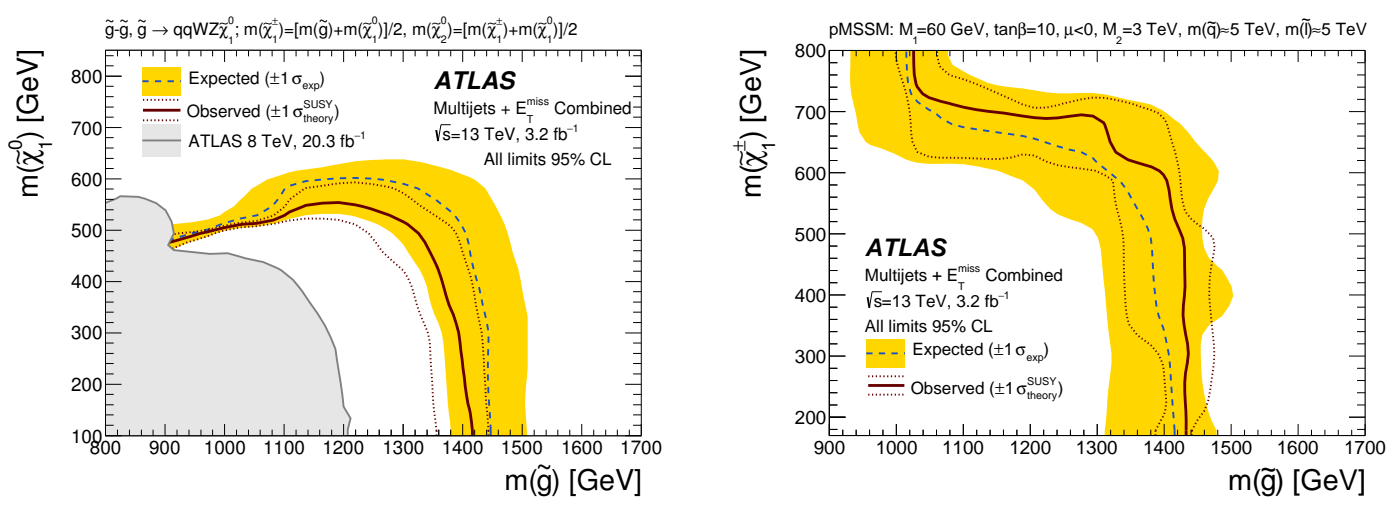

Figure 4: $95 \%$ CL exclusion curve for the '2-step' model (right) and the 'pMSSM' model (left) described in the text. The solid red and dashed blue curves show the 95\% CL observed and expected limits, respectively, including all uncertainties except the theoretical signal cross-section uncertainty (PDF and scale). The dotted red lines bracketing the observed limit represent the result produced when moving the signal cross-section by $\pm 1 \sigma$ (as defined by the PDF and scale uncertainties). The shaded yellow band around the expected limit shows the $\pm 1 \sigma$ variation of the expected limit. Excluded regions are below and to the left of the relevant lines. [4]

between the gluino and the $\widetilde{\chi}_{1}^{0}$. The Gtt model can be divided into two channels, one with hadronically decaying $\mathrm{W}$ bosons and the other where at least one of the $\mathrm{W}$ bosons decays leptonically, the 0 -lepton and the 1-lepton channel respectively. The 0-lepton channel vetoes any signal leptons and requires at least eight signal jets, $\Delta \Phi\left(\right.$ jet, $\left.E_{\mathrm{T}}^{\text {miss }}\right)>0.4$ and a minimum transverse mass of $E_{\mathrm{T}}^{\text {miss }}$ and any of the leading three b-jets $m_{\mathrm{T} \text {, min }}^{\text {bjets }}$ larger than $80 \mathrm{GeV}$. The transverse mass $m_{\mathrm{T}}$ is generally calculated as $m_{\mathrm{T}}=\sqrt{2 p_{\mathrm{T}}^{X} E_{\mathrm{T}}^{\text {miss }}\left\{1-\cos \left[\Delta \Phi\left(\vec{p}_{\mathrm{T}}^{\text {miss }}, X\right)\right]\right\}}$ with $X$ the respective particle. As for the Gbb SRs there are varying selections applied for different mass splittings for $E_{\mathrm{T}}^{\mathrm{miss}}$ and $m_{\mathrm{eff}}$ (incl.) . In addition the requirement on the number of $b$-jets is increased with decreasing mass splitting for the Gtt 0-lepton channel. For moderate and large mass splitting the top quarks tend to be boosted so the 0-lepton channels requires at least on top-tagged large-radius jet (using anti- $k_{t}$ with $\mathrm{R}=1.0$ ) for these SRs. The Gtt 1-lepton channel has two SRs only selecting events with at least one signal lepton, more than six jets of which three need to be b-tagged and $m_{\mathrm{T}}>150 \mathrm{GeV}$ calculated from the lepton and $E_{\mathrm{T}}^{\text {miss }}$ as well as $m_{\mathrm{T} \text {, min }}^{\text {b-jets }}>160 \mathrm{GeV}$. One SR is targeting large mass splittings by requiring in addition at least on top-tagged large-radius jet. This selection is not applied on the SR aiming at moderate to small mass splittings. Again the requirements for $E_{\mathrm{T}}^{\mathrm{miss}}$ and $m_{\mathrm{eff}}$ (incl.) are varied for different mass splittings.

The dominant background for all SRs is $t \bar{t}$ production in association with jets, it is estimated via a simultaneous fit of CRs orthogonal to the corresponding SRs. The resulting background prediction is cross-checked in VRs that are kinematically close to the SRs . The sub-dominant backgrounds like $t \bar{t}$ production together with a W/Z/h boson, single top or W/Z+jets are estimated directly from MC simulation normalized to data. For the estimation of the multijet background it is found that its contribution is negligible.

The results agree well with the SM. They are interpreted as model-independent limits on the visible cross-section and as exclusion limits in the models' phase spaces. The model-dependent fit of the Gbb model uses the SR with the best expected sensitivity for each point in the phase space. For the 
Gtt model the 0-lepton and 1-lepton channel are statistically combined in a simultaneous fit. For the simplified Gbb models gluino masses below $1.78 \mathrm{TeV}$ can be excluded at $95 \%$ confidence level for LSP masses below $700 \mathrm{GeV}$. For a massless lightest neutralino gluino masses below $1.76 \mathrm{TeV}$ are excluded in the Gtt model. The limits are shown in figure 5.
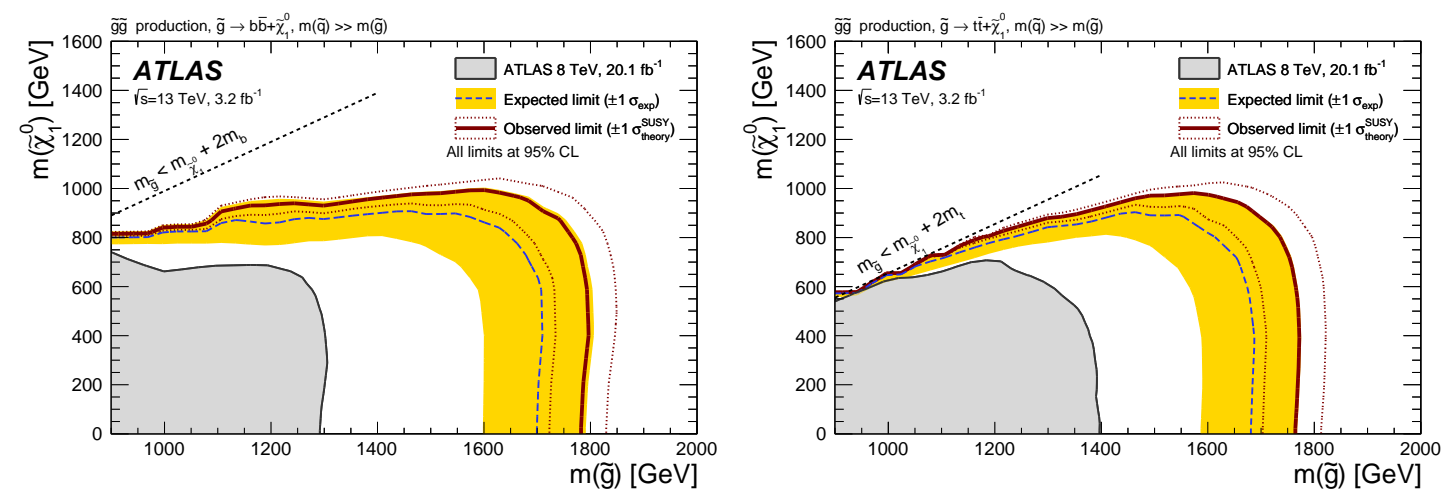

Figure 5: Exclusion limits in the $\widetilde{\chi}_{1}^{0}$ and $\widetilde{g}$ mass plane for the Gbb and Gtt models. The dashed and solid bold lines show the 95\% CL expected and observed limits, respectively. The shaded bands around the expected limits show the impact of the experimental and background theoretical uncertainties. The dotted lines show the impact on the observed limit of the variation of the nominal signal cross-section by $\pm 1 \sigma$ of its theoretical uncertainty. [5]

\section{Di-photon search}

The search for supersymmetry in a final state containing two photons and missing transverse momentum is tailored to look for general gauge mediation (GGM) models of SUSY. In GGM models the LSP is the Gravitino $\widetilde{G}$. The search results are interpreted in a model where the gluino decays via a $q \bar{q}$ pair and a $\widetilde{\chi}_{1}^{0}$ to the gravitino and a photon. The branching fraction to a photon and the $\widetilde{G}$. is dominant for all neutralino masses, for a massless $\widetilde{\chi}_{1}^{0}$ it becomes $100 \%$.

There is only one SR covering both high and low neutralino mass spectra. The optimization process showed that the selection criteria for both cases are very similar. Events are required to pass the following selection: two photons with a $\mathrm{p}_{\mathrm{T}}$ larger than $75 \mathrm{GeV}, E_{\mathrm{T}}^{\text {miss }}>175 \mathrm{GeV}, m_{\mathrm{eff}}>1500$ $\mathrm{GeV}$ and $\Delta \Phi\left(\right.$ jet, $\left.E_{\mathrm{T}}^{\text {miss }}\right)>0.5 . m_{\mathrm{eff}}$ is the scalar sum of the signal photons $\mathrm{p}_{\mathrm{T}}$ and $E_{\mathrm{T}}^{\text {miss }}$. The $\Delta \Phi$ requirement is only applied if there are at least two jets with $\mathrm{p}_{\mathrm{T}}>75 \mathrm{GeV}$ in the event.

The main backgrounds for diphoton events are $\gamma \gamma+$ jets, 'jet-faking-photons' from $\gamma+$ jets and multijet production, 'electron-faking-photons' coming from leptonically decaying W/Z bosons and $t \bar{t}$ in association with jets and an irreducible background from $W \gamma \gamma$ and $Z \gamma \gamma$ events. The real background from $\gamma \gamma+$ jets is estimated from MC simulation. For the estimation of the fake photon backgrounds a fake-factor method is used with CRs enriched with background events. Selected fake photon events due to jets and electrons are weighted with the jet fake-factor and the electron fake-factor respectively. The background estimation for $Z \gamma \gamma$ is taken directly from MC simulation. The same approach is used for $W \gamma \gamma$ but in addition the estimate is normalized to data in a dedicated l $\gamma \gamma \mathrm{CR}$.

The result shows no significant excess above the SM expectation. Figure 6 shows the model- 
dependent limit for the GGM model obtained by a simultaneous fit of the SR and the $W \gamma \gamma \mathrm{CR}$. For all possible LSP masses gluino masses below $1650 \mathrm{GeV}$ can be excluded at $95 \%$ confidence level.

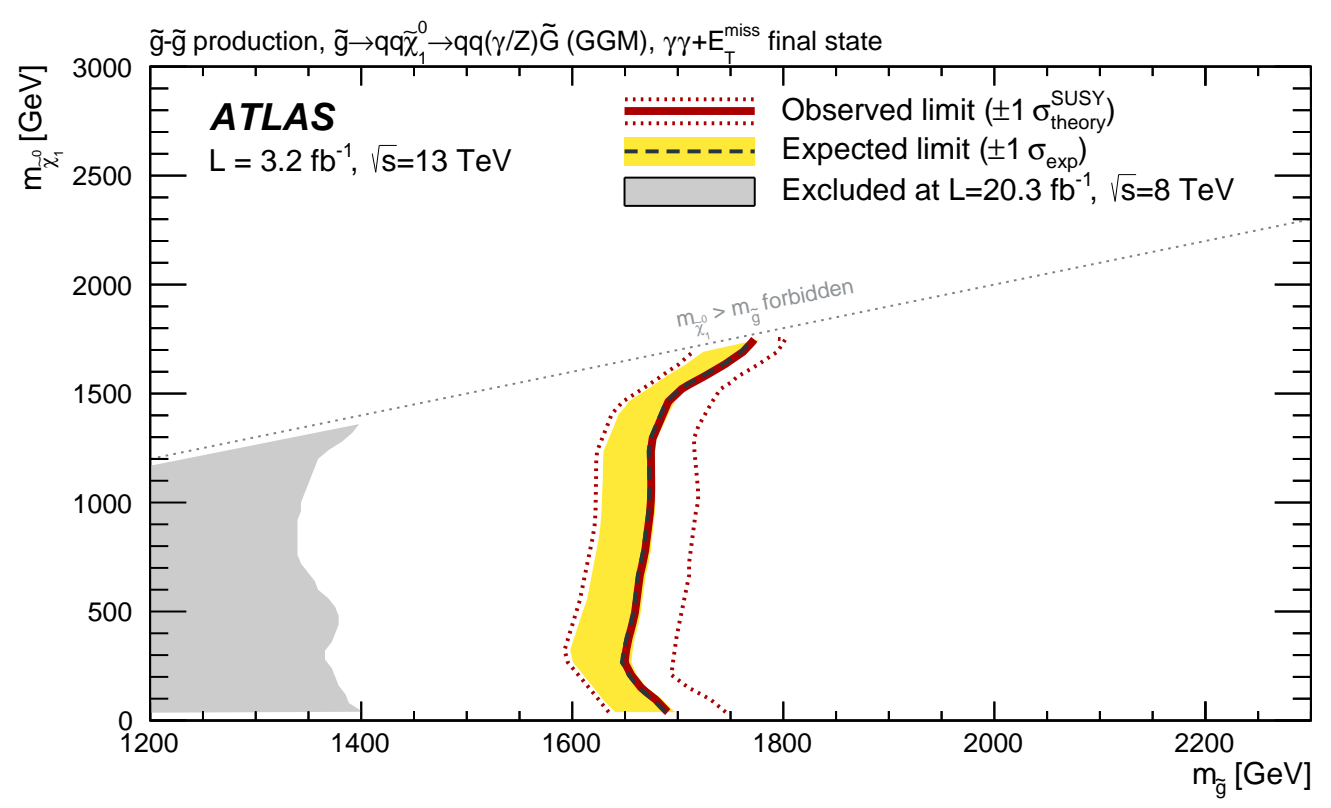

Figure 6: Exclusion limits in the neutralino-gluino mass plane at 95\% CL. The observed limits are exhibited for the nominal SUSY model cross section, as well as for a SUSY cross section increased and lowered by one standard deviation of the cross-section systematic uncertainty. Also shown is the expected limit, as well as the \pm 1 standard-deviation range of the expected limit, which is asymmetric because of the low count expected. Because the background expectation is close to zero and the observed number of events is zero, the expected and observed limits nearly overlap. [6]

\section{Conclusions}

Four ATLAS SUSY searches have been presented looking for the dominant strong production of squarks and gluinos. Each search is targeting different and well motivated SUSY models and therefore final states. In all cases no significant excess above Standard Model expectation was observed. With $3.2 \mathrm{fb}^{-1}$ of analyzed data at the LHC with $\sqrt{s}=13 \mathrm{TeV}$ in 2015 the existing limits on squark and gluino masses could be increased by all four searches. Figure 7 shows all limits with $3.2 \mathrm{fb}^{-1}$ of data for strongly produced squarks and gluinos. Depending on the models investigated in the included searches gluino masses below $1400 \mathrm{GeV}$ up to $1.78 \mathrm{TeV}$ and squark masses below $1.03 \mathrm{TeV}$ are excluded for models with the LSP the lightest neutralino. In the GGM model with the gravitino as LSP gluino masses below $1.65 \mathrm{TeV}$ are excluded at $95 \%$ confidence level. Despite no observed excess the SUSY searches will be continued. The full 2016 dataset of $20-30 \mathrm{fb}^{-1}$ projected data offer new discovery opportunities.

\section{References}

[1] ATLAS Collaboration, The ATLAS Experiment at the CERN Large Hadron Collider, JINST 3 (2008) S08003 


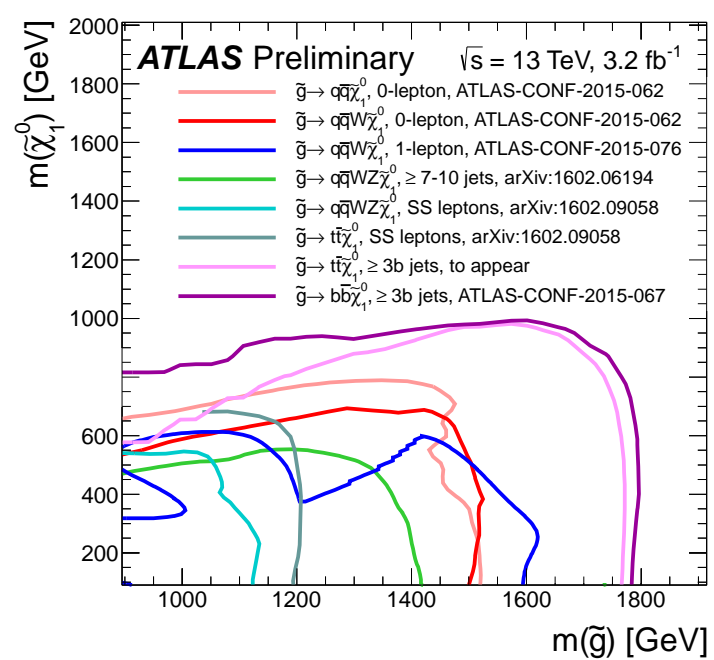

Figure 7: Exclusion limits at 95\% CL for $13 \mathrm{TeV}$ in the (gluino, lightest neutralino) mass plane for different simplified models featuring the decay of the gluino to the lightest neutralino either directly or through a cascade chain featuring other SUSY particles with intermediate mass. For each line, the gluino decay mode is reported in the legend and it is assumed to proceed with $100 \%$ branching ratio. [7]

[2] E. Halkiadakis, G. Redlinger and D. Shih, Status and Implications of Beyond-the-Standard-Model Searches at the LHC, Ann. Rev. Nucl. Part. Sci. 64 (2014) 319, [arXiv:1411.1427 [hep-ex]].

[3] ATLAS Collaboration, Search for squarks and gluinos in final states with jets and missing transverse momentum at $\sqrt{s}=13 \mathrm{TeV}$ with the ATLAS detector, Eur. Phys. J. C 76 (2016) 7:392, [arXiv:1605.03814 [hep-ex]].

[4] ATLAS Collaboration, Search for new phenomena in final states with large jet multiplicities and missing transverse momentum with ATLAS using $\sqrt{s}=13$ TeV proton-proton collisions, Phys. Lett. B 757 (2016) 334, [arXiv:1602.06194 [hep-ex]].

[5] ATLAS Collaboration, Search for pair production of gluinos decaying via stop and sbottom in events with $b$-jets and large missing transverse momentum in pp collisions at $\sqrt{s}=13 \mathrm{TeV}$ with the ATLAS detector, Phys. Rev. D 94 (2016) 032003, [arXiv:1605.09318 [hep-ex]].

[6] ATLAS Collaboration, Search for supersymmetry in a final state containing two photons and missing transverse momentum in $\sqrt{s}=13 \mathrm{TeV}$ pp collisions at the LHC using the ATLAS detector, [arXiv:1606.09150 [hep-ex]].

[7] https://twiki.cern.ch/twiki/bin/view/AtlasPublic/SupersymmetryPublicResults 